\title{
Leukemia Inhibitory Factor Receptor Is Involved in Apoptosis in Rat Astrocytes Exposed to Oxygen-Glucose Deprivation
}

\author{
Liang Huo $(\mathbb{D}$, Yuying Fan $(\mathbb{D}$, and Hua Wang $(\mathbb{D}$ \\ Department of Pediatrics, Shengjing Hospital of China Medical University, Shenyang, China \\ Correspondence should be addressed to Hua Wang; wangh1@sj-hospital.org
}

Received 12 January 2019; Accepted 12 February 2019; Published 27 February 2019

Guest Editor: Hengjia Ni

Copyright (C) 2019 Liang Huo et al. This is an open access article distributed under the Creative Commons Attribution License, which permits unrestricted use, distribution, and reproduction in any medium, provided the original work is properly cited.

Leukemia inhibitory factor (LIF) and leukemia inhibitory factor receptor (Lifr) protect CNS cells, specifically neurons and myelinsheath oligodendrocytes, in conditions of oxygen-glucose deprivation (OGD). In the case of astrocyte apoptosis resulting from reperfusion injury following hypoxia, the function of the Lifr remains to be fully elucidated. This study established models of in vivo ischemia/reperfusion (I/R) using an in vitro model of OGD to investigate the direct impact of silencing the Lifr on astrocyte apoptosis. Astrocytes harvested from newborn Wistar rats were exposed to OGD. Cell viability and apoptosis levels were determined by the MTT (3-(4,5-dimethylthiazol-2-yl)-2,5-diphenyltetrazolium bromide) assay and annexin V/propidium iodide (PI) staining assays, respectively. Apoptosis was further investigated by the TdT-mediated dUTP nick-end labelling (TUNEL) assay. A standard western blotting protocol was applied to determine levels of the protein markers Bcl2, Bax, p-Akt/Akt, p-Stat3/Stat3, and p-Erk/Erk. The cell viability assay (MTT) showed that astrocyte viability decreased in response to OGD. Furthermore, blocking RNA to silence the Lifr further reduces astrocyte viability and increases levels of apoptosis as detected by annexin V/PI double staining. Likewise, western blotting after Lifr silencing demonstrated increased levels of the apoptosis-related proteins Bax and p-Erk/Erk and correspondingly lower levels of $\mathrm{Bcl} 2$, p-Akt/Akt, and p-Stat/Stat3. The data gathered in these analyses indicate that the Lifr plays a pivotal role in the astrocyte apoptosis induced by hypoxic/low-glucose environments. Further investigation of the relationship between apoptosis and the Lifr may provide a potential therapeutic target for the treatment of neurological injuries.

\section{Introduction}

Hypoxic-ischemic encephalopathy (HIE) is known to be a major cause of child mortality and disability, but the processes that lead to neuronal apoptosis in HIE are as yet undefined. Previous research has focused on blood flow and vasculature and the effects on neurons [1,2]; however, interest has grown in the pathophysiology of astrocytes and their role in HIErelated conditions [3]. Astrocytes are the glial cell type present in the greatest numbers within the brain; they repair and maintain brain tissues and provide trophic, structural, and metabolic support to neurons and facilitate formation of neuronal synapses [4-6]. Given the pivotal role of astrocytes in CNS metabolism and glutamate balance, their death or disruption results in damage to the CNS and neuronal cell death $[7,8]$. It is recognised that astrocytes are involved in many events resulting from cerebral ischemia and hypoxiaischemia (HI) $[9,10]$. Specifically, early astrocyte death due to hypoxia and ischemia leads to an interruption in key mechanisms, generating greater neuronal apoptosis, which causes larger lesions and disrupts synaptogenesis [11, 12]. In light of their critical role, it is clear that astrocytes offer a robust therapeutic target to minimise damage resulting from cerebral HI.

Leukemia inhibitory factor (LIF) belongs to an interleukin 6 (IL-6) class and is recognised as a neuroprotector with anti-inflammatory properties $[13,14]$. Furthermore, acting on macrophages and T-helper cells, LIF induces an antiinflammatory phenotype [15]. Production of LIF is stimulated 
by the proinflammatory cytokines IL- 6 and tumor necrosis factor- $\alpha$. The beneficial functions of LIF are demonstrable both histologically and functionally, depending on the maturity and the cell type upon which it acts. Activation of the LIF receptor (Lifr), a 190-kD type 1 cytokine receptor located in the nuclei of neuronal cells until injury occurs, initiates upon binding of LIF [16-18]. Protein-receptor binding forms a high-affinity complex that starts the LIF signal through several cellular pathways, including Janus kinase- (JAK-) signal transducer and activator of transcription (STAT), mitogenactivated protein kinase (MAPK), and extracellular signalregulated kinase (ERK), preserving stroke-damaged brain cells [19]. Additionally, LIF/Lifr binding drives phenotypic alterations in T cells and macrophages to switch to an antiinflammatory response from the immediate inflammatory response to brain injury, which leads to neurodegeneration $[20,21]$. The signal-transducing role of the Lifr is widely recognised, but its molecular and cellular functions remain to be elucidated.

This research aimed to define the role of Lifr in OGD and the mechanism by which it affects hypoxic-ischemic astrocytes. To accomplish this goal, the impact of RNA disruption of Lifr on apoptosis levels in primary rat astrocyte cultures was assessed. To gain deeper insight into the function of the Lifr in astrocytes deprived of oxygen and glucose, expression levels of a range of apoptosis-related proteins were determined. The proteins assessed included Lifr, B-cell lymphoma 2 (Bcl2), Bax, p-Akt/Akt, p-Stat3/Stat3, and pErk/Erk.

\section{Materials and Methods}

2.1. Primary Culture of Astrocytes. The Local Animal Ethics Committee of China Medical University approved the protocols and procedures for care and use of animals in this research. Primary astrocyte cultures were derived from newborn Wistar rats. Briefly, the cerebral cortices were harvested following hypothermic anaesthetisation and decapitation. After excision of the meninges, the cortical tissue was cut into small pieces and added to culture medium. The tissue suspension was mixed by vortexing and then filtred through nylon mesh filtres, firstly of pore size $80 \mu \mathrm{m}$ and then $10 \mu \mathrm{m}$. The resulting filtrate was further diluted in Dulbecco's minimal essential medium (DMEM) with $7.5 \mathrm{mM}$ glucose and $20 \%$ horse serum. The cultures were placed in a humidified incubator at $37^{\circ} \mathrm{C} / 5 \% \mathrm{CO}_{2}$. Cultures were maintained by exchanging medium with $10 \%$ serum on day 3 and thereafter every 3-4 days. Rotary shaking of the culture flasks at $260 \mathrm{rpm}$ facilitated the removal of other cell types. Primary astrocytes were passaged a maximum of three times in culture and used in assays when $80-90 \%$ confluent. The astrocyte cultures showed a near-uniform immunoreactivity (greater than 95\%) to glial fibrillary acidic protein.

2.2. Hypoxia and Glucose Deprivation Experiments. Briefly, following a wash with glucose-free culture medium, primary astrocyte cultures were supplied with fresh glucosefree medium in the absence of serum. For the hypoxic conditions, the cells were placed in a humidified Tri-gas incubator (Thermo Scientific, Waltham, MA) for 24 hours, with conditions of $94 \% \mathrm{~N}_{2} / 5 \% \mathrm{CO}_{2} / 1 \% \mathrm{O}_{2}$. Following the 24-hour incubation, the cells were returned to standard incubator conditions (see Section 2.1) for an additional 24 hours. Control cells, used in all assays, were maintained in normal incubator conditions and fed with glucose-containing DMEM.

2.3. RNA Interference. Transient transfection of small interfering RNAs (SiRNAs; Jima Medicine, Shanghai, China) was conducted using lipofectamine reagent (Invitrogen, Grand Island, NY) on 70\% confluent primary astrocytes in six-well plates in accordance with the manufacturer's instructions. The sequences of the SiRNAs used to interrupt expression of Lifr were as follows: sense $5^{\prime}$ GGUGAUCACGAAGUAACAATT- $3^{\prime}$ and antisense $5^{\prime}$ UUGUUACUUCGUGAUCACCTT-3'. Western blotting was used to demonstrate effective downregulation of Lifr expression.

2.4. Cell Viability and Apoptosis Assays. MTT assays were conducted using a kit purchased from Sigma (USA) to determine cell viability. To determine levels of apoptosis, cells were double-stained with annexin V/PI in accordance with manufacturer's protocols, and cells stained positively were enumerated by flow cytometry (FACSCalibur ${ }^{\mathrm{TM}}$, Becton Dickinson, San Jose, CA). Data were analysed by CellQuest ${ }^{\mathrm{TM}}$ software (BD Biosciences). To summarise, the apoptosis protocol was conducted on astrocytes harvested in ice-cold PBS and pelleted by centrifugation. Thereafter, cells were rinsed in staining buffer and exposed to $100 \mu \mathrm{l}$ of buffer containing $5 \mu \mathrm{l}$ of annexin V-FITC and $5 \mu \mathrm{g} / \mathrm{ml}$ of PI, for 30 minutes at $4^{\circ} \mathrm{C}$ in darkness. Following incubation, the cells were washed and resuspended in $250 \mu \mathrm{l}$ of staining buffer prior to flow cytometry analysis using both forward- and side-scatter light. CellQuest software was used to assess the output from 10,000 events. Apoptotic cells are those that exhibit positive annexin V-FITC staining in combination with negative PI staining.

2.5. TdT-Mediated dUTP Nick-End Labelling (TUNEL) Assay. The cells were stained with the In Situ Cell Death Detection Kit (Cat. No. 11684817910, Roche), in accordance with the manufacturer's recommended protocol. Cell nuclei were stained with $4^{\prime}$,6-diamidino-2-phenylindole (DAPI, Roche), and the cells were visualised by fluorescent microscopy (Olympus iX70). Levels of apoptosis were calculated as a percentage of positive cells per 1000 DAPI-stained nuclei.

2.6. Western Blotting. Astrocyte cultures were prepared for western blotting as described by others [22], with $30 \mu \mathrm{g}$ of protein (unless otherwise noted) being separated by $10 \%$ SDS-PAGE. The gel-separated proteins were then electroblotted to transfer to a polyvinylidene difluoride membrane. The transferred proteins were probed with the following primary antibodies: Anti-LIFR antibody (ab202847, Abcam, 1:1000), Beta Actin Mouse Monoclonal antibody (66009-1-ig, Proteintech, 1:5000), Phospho-AKT (Ser473) (D9E)XP Rabbit mAb (Cell Signaling Technology, 1:2000), 
AKT (pan) (C67E7) Rabbit mAb (Cell Signaling Technology, 1:1000), Phospho-Stat3 (Tyr705) (D3A7) XP Rabbit mAb (Cell Signaling Technology, 1:2000), Stat3 (D3Z2G) Rabbit mAb (Cell Signaling Technology, 1:1000), Anti-Bcl2 Rabbit mAb (ab196495, Abcam, 1:1000), Anti-Bax Rabbit mAb (ab32509, Abcam, 1:2000), Phospho-p44/42 MAPK (Erk1/2) (Thr202/Tyr204) (D13.14.4E) XP ${ }^{\circledR}$ Rabbit mAb (Cell Signaling Technology, 1:2000), and p44/42 MAPK (Erk1/2) (137F5) Rabbit mAb (Cell Signaling Technology, 1:1000). The membrane was washed and then incubated with the secondary antibodies HRP-conjugated Affinipure Goat Anti-Rabbit IgG(H+L) (SA00001-2, Proteintech, 1:2000) and HRP-conjugated Affinipure Mouse Anti-Rabbit IgG $(\mathrm{H}+\mathrm{L})$ (SA00001-1, Proteintech, 1:2000). After another wash, the detection substrate Immobilon ${ }^{\mathrm{TM}}$ Western Chemiluminescent HRP Substrate (Millipore) was applied.

2.7. Statistical Analysis. Statistical analysis ( $t$-test) was conducted using GraphPad Prism V.7.0, from mean data ( \pm SEM) derived from three independent tests. Statistical significance was determined as $P<0.05$.

\section{Results}

The efficacy of the Lifr knockdown was measured by western blotting of the LIFR expressed protein. The levels of LIFR were significantly lower $(P<0.05)$ in both the ORNA-silenced groups (N+SiRNA) and oxygen-glucose deprivation (OGD) plus SiRNA (OGD+SiRNA) groups than in either control (normal control, N) or ODG treatment group (Figure 1). Furthermore, the levels of LIFR protein in the OGD treatment group were significantly higher than in the normal control $(\mathrm{N})$ group $(P<0.05)$ (Figure 1$)$.

Astrocyte cell viability was determined by the MTT assay (Figure 2). OGD-treated cells had significantly lower $(P<0.01)$ levels of cell viability, showing a $23 \%$ reduction in comparison with the $\mathrm{N}$ controls. Silencing of the Lifr in otherwise normal cells led to a slight reduction in cell viability. However, silencing of the Lifr in OGD-treated cells revealed a significant $(P<0.05)$ decrease in viability compared with OGD-treated cells. These data indicate that under OGD conditions, silencing of the Lifr significantly decreased cell viability and stimulated cell damage.

Next, we determined the effect of Lifr silencing on apoptosis levels of OGD-treated astrocytes, using the annexin V/PI double-staining method (Figure 3). The results show a significant increase $(P<0.01)$ in apoptosis levels in both OGD-treated and SiRNA+OGD-treated groups in comparison with $\mathrm{N}$ and $\mathrm{N}+\mathrm{SiRNA}$, respectively. Furthermore, apoptosis increased significantly $(P<0.05)$ in both the silenced $(\mathrm{N}+\mathrm{SiRNA})$ group in comparison with the $\mathrm{N}$ group and the OGD+SiRNA group in comparison with the OGD group.

Levels of apoptosis were further elucidated by testing each group with the TUNEL assay (Figure 4). N and N+SiRNA astrocytes demonstrated low numbers of apoptotic cells, whereas both OGD and OGD-SiRNA astrocytes exhibited large numbers of apoptotic cells. Silencing of both the N cells $(\mathrm{N}+\mathrm{SiRNA})$ and OGD-treated cells (OGD+SiRNA) led to a

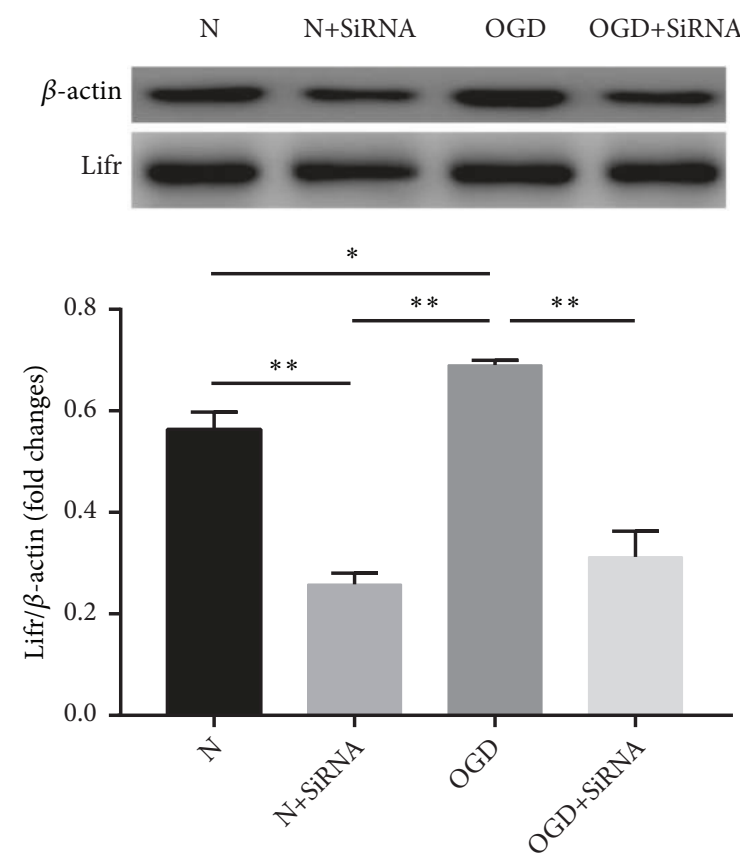

FIGURE 1: LIFR levels in response to SiRNA treatment in comparison with untreated control and OGD-treated cells. Error bars represent mean \pm SEM $(* P<0.05 ; * * P<0.01)$.

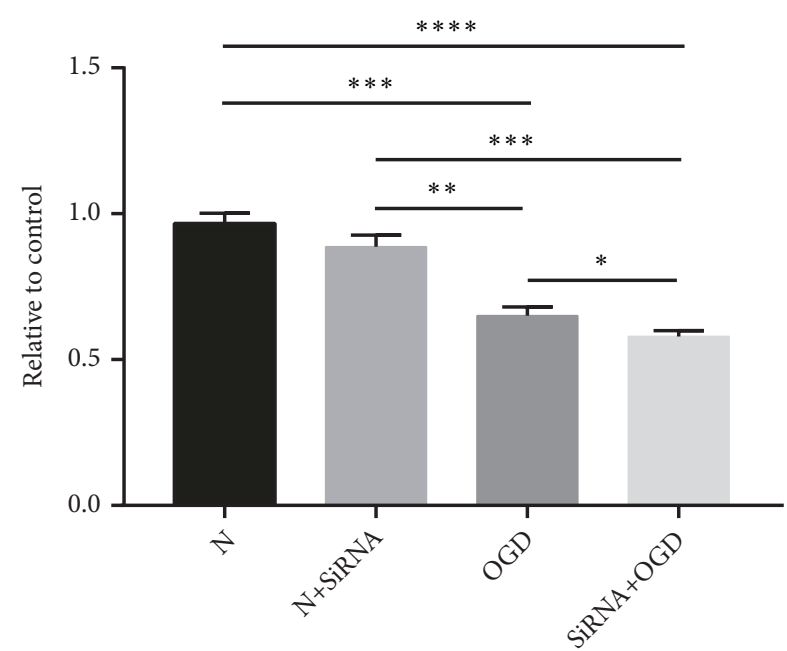

FIGURE 2: Comparison of cell viability in normal, normal-silenced, OGD-treated, and OGD-treated and silenced astrocytes as determined by MTT assay. Error bars represent mean \pm SEM $(* P<0.05$; $* * P<0.01 ; * * * P<0.001)$.

significant increase in apoptotic cells compared with $\mathrm{N}$ and OGD cells, respectively $(P<0.05$ and $P<0.001$, respectively). These data suggest that suppression of Lifr further stimulates OGD-induced apoptosis in primary astrocytes.

Levels of proteins in astrocytes that are associated with apoptosis (i.e., B-cell lymphoma 2 ( $\mathrm{Bcl} 2$ ), BAX, p-Akt/Akt, pStat3/Stat3, and p-Erk/Erk) were assessed by western blotting (Figure 5). OGD treatment led to a significant reduction $(P$ $<0.01$ ) in levels of $\mathrm{Bcl} 2$ in comparison with the $\mathrm{N}$ group, whereas silencing of OGD-treated cells further rescued these 

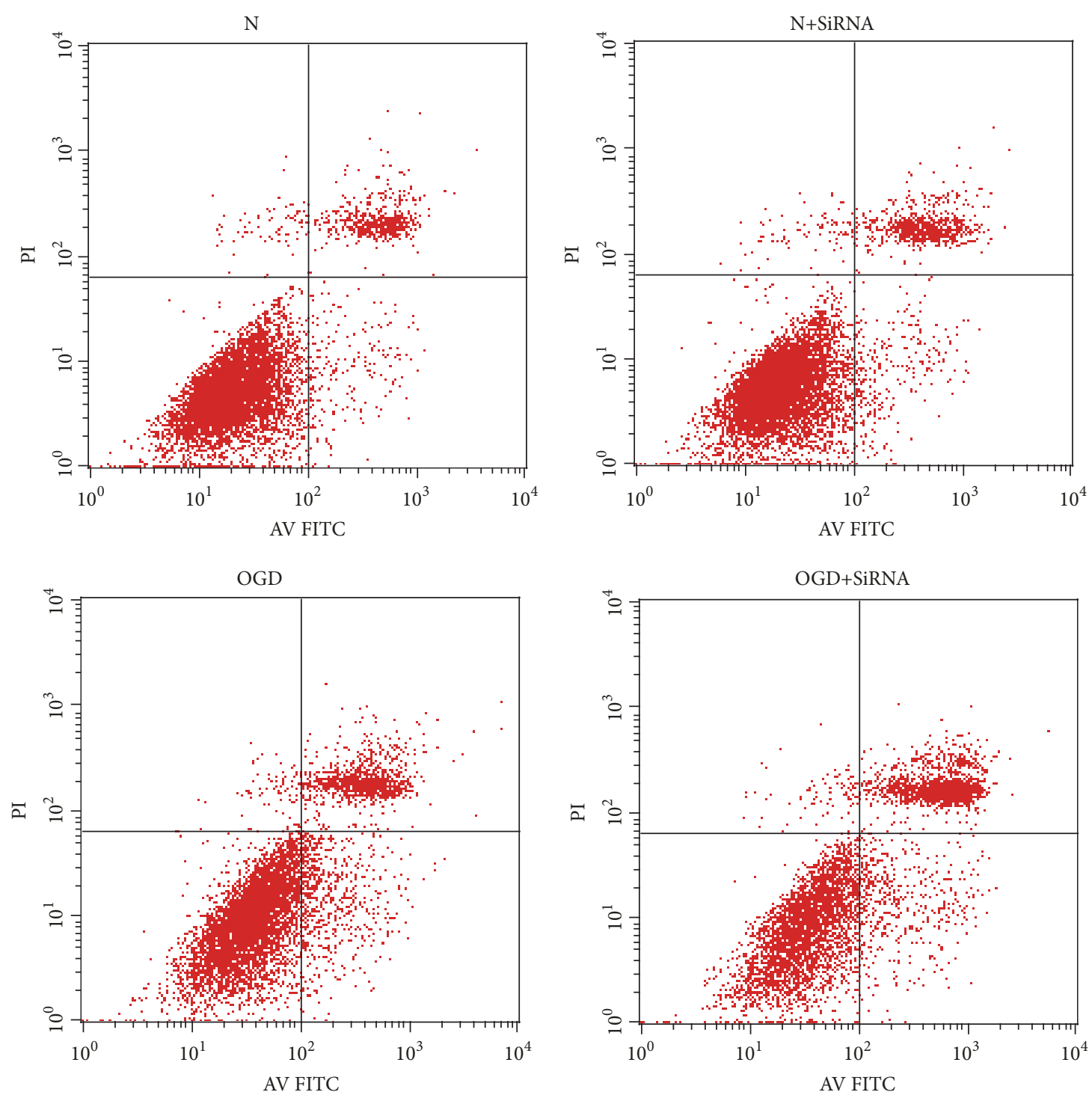

(a)

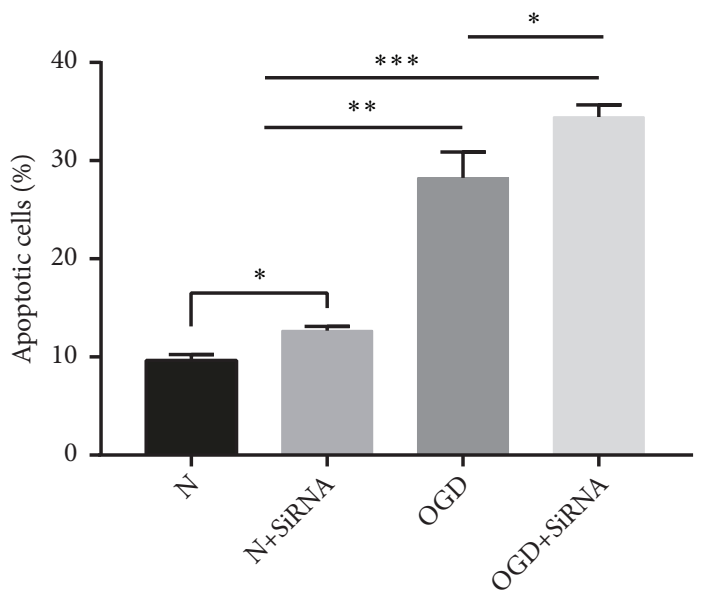

(b)

FIGURE 3: Comparison of apoptosis levels in normal, normal-silenced, OGD-treated, and OGD-treated and silenced astrocytes using annexin V/PI double staining $(* P<0.05 ; * * P<0.01 ; * * * P<0.001)$. 

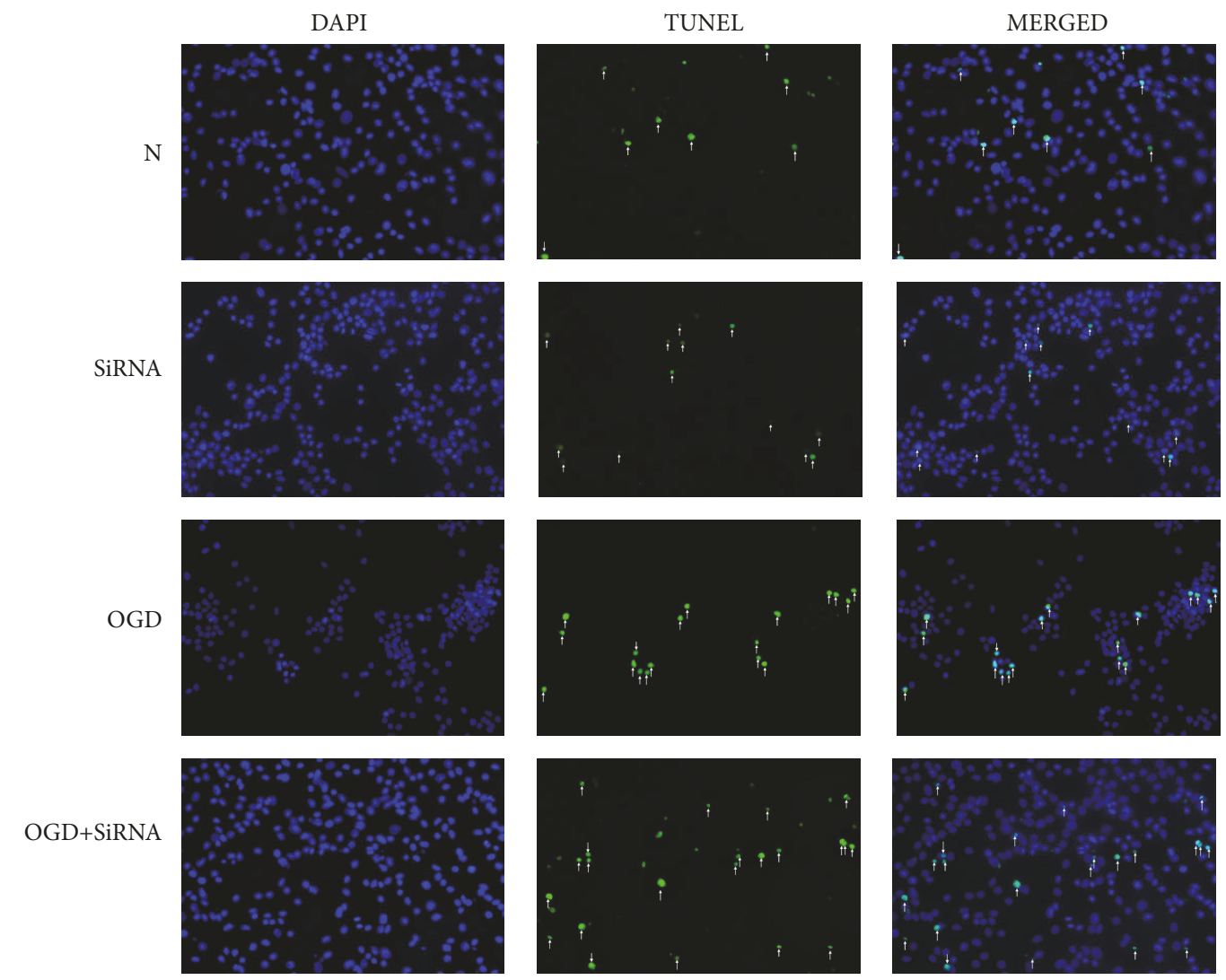

(a)

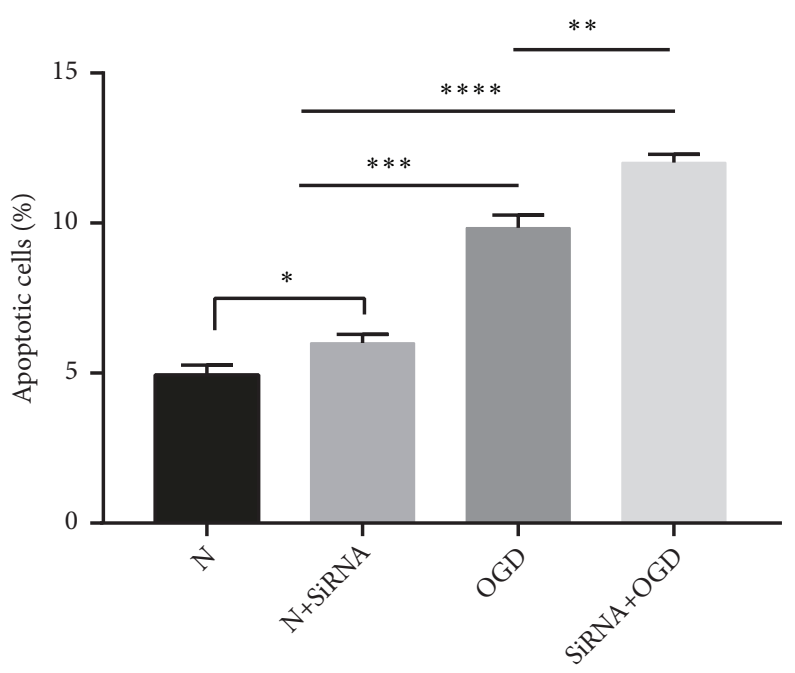

(b)

FIGURE 4: Apoptosis rates in normal, normal-silenced, OGD-treated, and OGD-treated and silenced astrocytes using the TUNEL assay $(\times 200$ magnification). Error bars represent mean \pm SEM $(* P<0.05 ; * * P<0.01 ; * * * P<0.001)$.

levels $(P<0.01)$ in comparison with OGD treatment $(P$ $<0.01)$. Correspondingly, levels of BAX were significantly higher in the OGD group than the $\mathrm{N}$ group $(P<0.01)$ and in the OGD-SiRNA group than in the OGD group $(P<0.05)$.

The ratios of the proteins $\mathrm{p}$-Akt/Akt and $\mathrm{p}$-Stat $3 /$ Stat3 were significantly lower in the OGD group compared with the $\mathrm{N}$ group $(P<0.05)$ and in the OGD-SiRNA group compared with the OGD group $(P<0.05)$. Conversely, ratios of $\mathrm{p}$ Erk/Erk increased significantly in both OGD $(P<0.05)$ and OGD+SiRNA $(P<0.01)$, compared with the $\mathrm{N}$ and OGD groups, respectively. In combination, these data indicate a critical role for these pathways in OGD-induced apoptosis, and silencing Lifr may regulate these proteins in a manner that further promotes apoptosis in OGD-treated cells. 


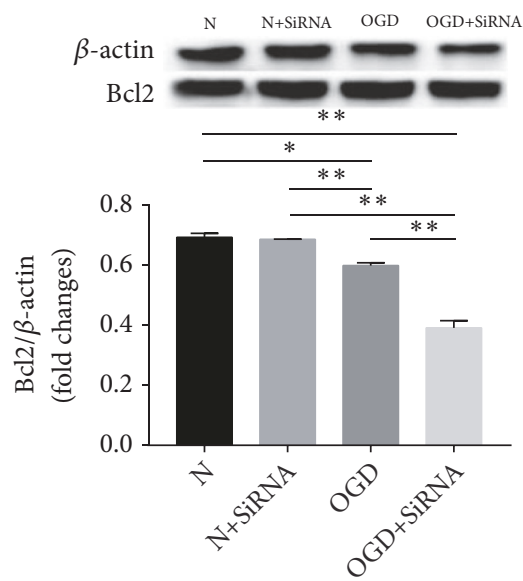

(a)

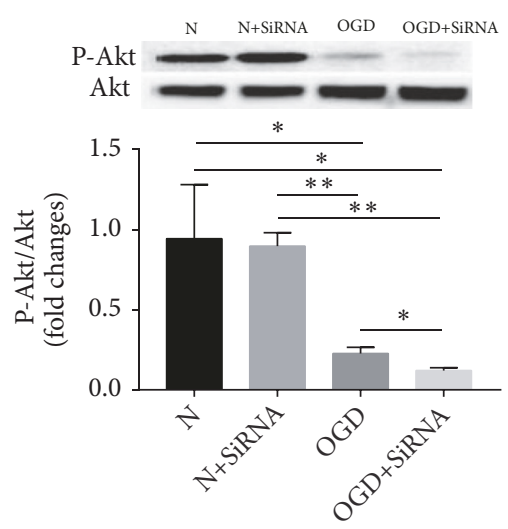

(d)

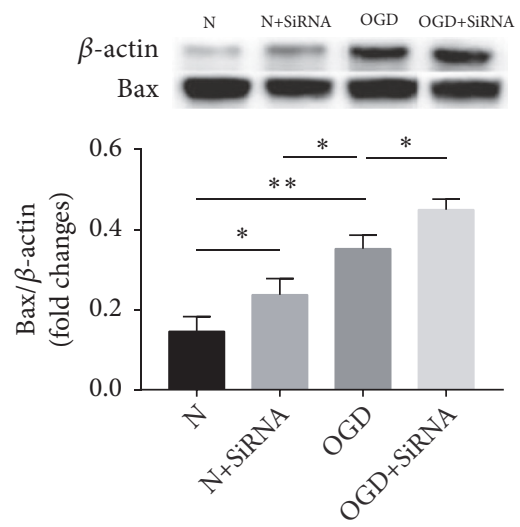

(b)

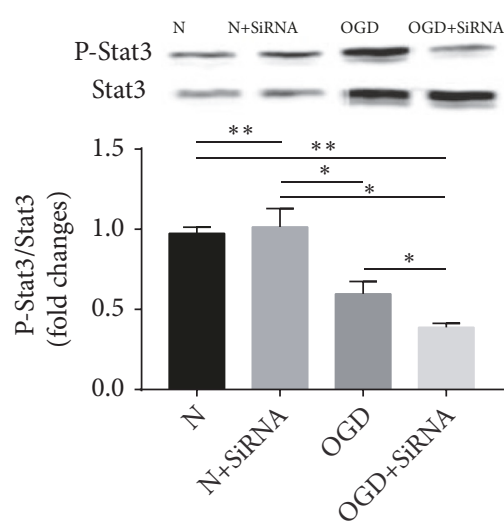

(e)

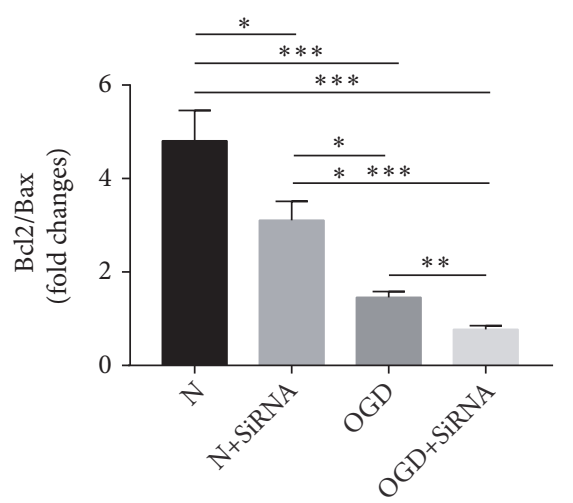

(c)

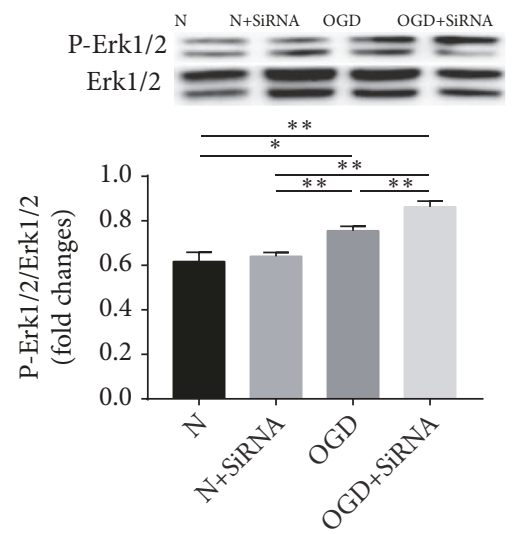

(f)

FIGURE 5: Signaling pathway in OGD-induced apoptosis of astrocytes was detected by western blotting $(* P<0.05 ; * * P<0.01)$.

\section{Discussion}

The link between brain injury resulting from ischemia and astrocyte damage is widely recognised [23, 24], and it is proposed here that protecting astrocytes in this environment from further apoptotic damage and death may be pivotal in partially protecting brain tissue from ischemic injury and thus improving postischemic functionality. OGD was used in vitro to induce apoptosis in astrocytes through two mechanisms: endoplasmic reticulum stress and mitochondrial disruption $[25,26]$. Our data show that cell viability, assessed by MMT, is reduced in OGD-treated astrocytes compared with normal (untreated) controls. Furthermore, silencing of the LIF receptor (Lifr) further decreases cell viability and increases apoptosis, as measured using the annexin V/PI method, in comparison with OGD-treated cells. Investigation of apoptosis pathways through western blotting analysis revealed significantly increased levels of $\mathrm{BAX}$ and $\mathrm{p}-\mathrm{ERK} / \mathrm{ERK}$ ratios, and a corresponding significant decrease in $\mathrm{Bcl} 2$ levels and in p-Akt/Akt and p-Stat3/Stat3 ratios in OGD+SiRNA-treated cells when compared with OGD-treated cells. These data indicate that Lifr control of apoptosis-signaling pathways is important in OGDstimulated astrocyte apoptosis.

The ratios of $\mathrm{Bcl}-2$ to $\mathrm{Bax}$ are intrinsically linked to the balance of apoptosis [27], as Bcl-2 is known as an antiapoptotic protein, the action of which is to prevent cellular apoptosis, whereas Bax is an apoptosis-stimulating protein $[27,28]$. A stable ratio of $\mathrm{Bcl}-2$ to $\mathrm{Bax}$ has been demonstrated to inhibit $\mathrm{H}_{2} \mathrm{O}_{2}$-induced apoptosis of cultured astrocytes. In apoptotic conditions, the action of Bax after translocation to the mitochondria enables the extracellular release of proapoptotic proteins and operates through permeabilisation of the cell membrane [28].

The protective effects of an increased $\mathrm{Bcl}-2 / \mathrm{Bax}$ ratio, through upregulation of $\mathrm{Bcl}-2$ and corresponding downregulation of Bax, was successfully demonstrated in a coculture of IL-6-producing mesenchymal stem cells and oxygenglucose deprived astrocytes, wherein the mesenchymal cells successfully inhibited apoptosis [29]. In our study, after OGD treatment, expression of Bcl-2 was decreased in comparison with controls and Bax was correspondingly increased, indicating an increased level of apoptosis. In OGD-treated 
astrocytes that were also exposed to silencing of Lifr, the ratio of Bcl-2 to Bax further decreased, indicating that, following $\mathrm{OGD}$, suppression of Lifr expression further reduces the Bcl2/Bax ratio and stimulates apoptosis.

Resulting from cell damage caused by several factors, apoptosis is a widely known cell death mechanism that is initiated by the upregulation of apoptotic protein production [27, 30-32]. Researchers have found that increased levels of Bax expression may lead to apoptosis via activation of procaspase-3 [32, 33], whereas others define the pivotal actions of $\mathrm{Bcl}-2, \mathrm{p}-\mathrm{Akt}$, and $\mathrm{p}-\mathrm{ERK} 1 / 2$ on balancing cell survival and death $[34,35]$. Suppression of Akt activity within the CNS is linked to post-HI injury-induced neuronal death, demonstrating the importance of the phosphoinositide 3kinase (PI3K)/Akt pathway as an antiapoptotic mechanism to protect neurons [36]. The activity of Akt is increased by phosphorylation of serine-473 (Ser473) $[37,38]$. We found in this study that p-Akt levels significantly decreased in response to I/R damage, with no effect on levels of unphosphorylated Akt. This response was further enhanced by silencing Lifr, whereby lower levels of p-Akt, corresponding to lower activity of Akt, were found. These data indicate that there is a role for Lifr in apoptosis regulation that may be managed via PI3K/Akt signaling.

Contributing to existing evidence of a neuroprotective role for Lifr, our data elucidate the impact of Lifr silencing on OGD-induced apoptosis. Furthermore, these data enhance our current knowledge of the biological function of Lifr and initiate a pathway for continued research from a clinical perspective to the applicability of Lifr in treating stroke and neurological damage. However, our work investigating the role of Lifr in neurological damage requires further investigation and expansion.

\section{Data Availability}

All the data are available from the correspondence author upon request.

\section{Conflicts of Interest}

The authors declare that there are no conflicts of interest regarding the publication of this article.

\section{Authors' Contributions}

Liang Huo and Yuying Fan contributed equally to this manuscript.

\section{Acknowledgments}

This work was supported by National Natural Science Foundation of China (81501299).

\section{References}

[1] F. Zhao, Y. Qu, H. Wang et al., "The effect of miR-30d on apoptosis and autophagy in cultured astrocytes under oxygenglucose deprivation," Brain Research, vol. 1671, pp. 67-76, 2017.
[2] B. J. Dixon, C. Reis, W. M. Ho, J. Tang, and J. H. Zhang, "Neuroprotective strategies after neonatal hypoxic ischemic encephalopathy," International Journal of Molecular Sciences, vol. 16, no. 9, pp. 22368-22401, 2015.

[3] D. Kasprowska, G. Machnik, A. Kost, and B. Gabryel, “Timedependent changes in apoptosis upon autophagy inhibition in astrocytes exposed to oxygen and glucose deprivation," Cellular and Molecular Neurobiology, vol. 37, no. 2, pp. 223-234, 2017.

[4] C. Farina, F. Aloisi, and E. Meinl, "Astrocytes are active players in cerebral innate immunity," Trends in Immunology, vol. 28, no. 3, pp. 138-145, 2007.

[5] C. Lambert, P. Cisternas, and N. C. Inestrosa, "Role of Wnt signaling in central nervous system injury," Molecular Neurobiology, vol. 53, no. 4, pp. 2297-2311, 2016.

[6] S. A. Liddelow, K. A. Guttenplan, L. E. Clarke et al., "Neurotoxic reactive astrocytes are induced by activated microglia," Nature, vol. 541, no. 7638, pp. 481-487, 2017.

[7] T. Chitnis and H. L. Weiner, "CNS inflammation and neurodegeneration," The Journal of Clinical Investigation, vol. 127, no. 10, pp. 3577-3587, 2017.

[8] A. Almad and N. J. Maragakis, "A stocked toolbox for understanding the role of astrocytes in disease," Nature Reviews Neurology, vol. 14, no. 6, pp. 351-362, 2018.

[9] Z. W. Liu and M. Chopp, "Astrocytes, therapeutic targets for neuroprotection and neurorestoration in ischemic stroke," Progress in Neurobiology, vol. 144, pp. 103-120, 2016.

[10] Y. Jin, S. Chen, N. Li et al., "Defect-related luminescent burlike hydroxyapatite microspheres induced apoptosis of MC3T3E1 cells by lysosomal and mitochondrial pathways," SCIENCE CHINA Life Sciences, vol. 61, no. 4, pp. 464-475, 2018.

[11] E. Ramos, P. Patiño, R. J. Reiter et al., "Ischemic brain injury: new insights on the protective role of melatonin," Free Radical Biology \& Medicine, vol. 104, pp. 32-53, 2017.

[12] E. Rocha-Ferreira and M. Hristova, "Plasticity in the neonatal brain following hypoxic-ischaemic injury," Neural Plasticity, vol. 2016, Article ID 4901014, 16 pages, 2016.

[13] S. M. Davis, L. A. Collier, C. C. Leonardo, H. A. Seifert, C. T. Ajmo Jr., and K. R. Pennypacker, "Leukemia inhibitory factor protects neurons from ischemic damage via upregulation of superoxide dismutase 3," Molecular Neurobiology, vol. 54, no. 1, pp. 608-622, 2017.

[14] D. D. Rowe, C. C. Leonardo, A. A. Hall et al., "Cord blood administration induces oligodendrocyte survival through alterations in gene expression," Brain Research, vol. 1366, pp. 172-188, 2010.

[15] K. Janssens, C. Van den Haute, V. Baekelandt et al., "Leukemia inhibitory factor tips the immune balance towards regulatory $\mathrm{T}$ cells in multiple sclerosis," Brain, Behavior, and Immunity, vol. 45, pp. 180-188, 2015.

[16] N. J. Gardiner, W. B. J. Cafferty, S. E. Slack, and S. W. N. Thompson, "Expression of gp130 and leukaemia inhibitory factor receptor subunits in adult rat sensory neurones: regulation by nerve injury," Journal of Neurochemistry, vol. 83, no. 1, pp. 100-109, 2002.

[17] S. M. Davis, L. A. Collier, S. Goodwin, D. E. Lukins, D. K. Powell, and K. R. Pennypacker, "Efficacy of leukemia inhibitory factor as a therapeutic for permanent large vessel stroke differs among aged male and female rats," Brain Research, vol. 1707, pp. 62-73, 2019.

[18] J.-W. Zhao, S. C. Dyson, C. Kriegel et al., "Modelling of a targeted nanotherapeutic 'stroma' to deliver the cytokine LIF, 
or XAV939, a potent inhibitor of Wnt- $\beta$-catenin signalling, for use in human fetal dopaminergic grafts in Parkinson's disease," Disease Models \& Mechanisms, vol. 7, no. 10, pp. 1193-1203, 2014.

[19] D. D. Rowe, C. C. Leonardo, J. A. Recio, L. A. Collier, A. E. Willing, and K. R. Pennypacker, "Human umbilical cord blood cells protect oligodendrocytes from brain ischemia through Akt signal transduction," The Journal of Biological Chemistry, vol. 287, no. 6, pp. 4177-4187, 2012.

[20] A. H. Miller and C. L. Raison, "The role of inflammation in depression: from evolutionary imperative to modern treatment target," Nature Reviews Immunology, vol. 16, no. 1, pp. 22-34, 2016.

[21] M. V. Russo and D. B. McGavern, "Immune surveillance of the CNS following infection and injury," Trends in Immunology, vol. 36, no. 10, pp. 637-650, 2015.

[22] B. Gabryel, A. Kost, D. Kasprowska et al., "AMP-activated protein kinase is involved in induction of protective autophagy in astrocytes exposed to oxygen-glucose deprivation," Cell Biology International, vol. 38, no. 10, pp. 1086-1097, 2014.

[23] N. Sun, J.-R. Hao, X.-Y. Li et al., "GluR6-FasL-Trx2 mediates denitrosylation and activation of procaspase-3 in cerebral ischemia/reperfusion in rats," Cell Death \& Disease, vol. 4, article e771, 2013.

[24] K. Zhu, Q. He, L. Li, Y. Zhao, and J. Zhao, "Silencing thioredoxinl exacerbates damage of astrocytes exposed to OGD/R by aggravating apoptosis through the Actin-Ras2-cAMP-PKA pathway," International Journal of Neuroscience, vol. 128, no. 6 , pp. 512-519, 2018.

[25] G. Faraco, S. Fossati, M. E. Bianchi et al., "High mobility group box 1 protein is released by neural cells upon different stresses and worsens ischemic neurodegeneration in vitro and in vivo," Journal of Neurochemistry, vol. 103, no. 2, pp. 590-603, 2007.

[26] Y. Y. Fan, J. M. Zhang, H. Wang, X. Y. Liu, and F. H. Yang, "Leukemia inhibitory factor inhibits the proliferation of primary rat astrocytes induced by oxygen-glucose deprivation," Acta Neurobiologiae Experimentalis, vol. 73, no. 4, pp. 485-494, 2013.

[27] L. Grosse, C. A. Wurm, C. Brüser, D. Neumann, D. C. Jans, and S. Jakobs, "Bax assembles into large ring-like structures remodeling the mitochondrial outer membrane in apoptosis," EMBO Journal, vol. 35, no. 4, pp. 402-413, 2016.

[28] Y. Liu, X. Zeng, Y. Hui et al., "Activation of $\alpha 7$ nicotinic acetylcholine receptors protects astrocytes against oxidative stress-induced apoptosis: implications for Parkinson's disease," Neuropharmacology, vol. 91, pp. 87-96, 2015.

[29] L. Yaidikar and S. Thakur, " Punicalagin attenuated cerebral ischemia-reperfusion insult via inhibition of proinflammatory cytokines, up-regulation of Bcl-2, down-regulation of Bax, and caspase-3," Molecular and Cellular Biochemistry, vol. 402, no. 12, pp. 141-148, 2015.

[30] Q. Jiang, J. Chen, S. Liu, G. Liu, K. Yao, and Y. Yin, "1Glutamine attenuates apoptosis induced by endoplasmic reticulum stress by activating the IRE1 $\alpha$-XBP1 axis in IPEC-J2: a novel mechanism of l-glutamine in promoting intestinal health," International Journal of Molecular Sciences, vol. 18, no. 12, 2017.

[31] X. Ying, A. Huang, Y. Xing, L. Lan, Z. Yi, and P. He, "Lycorine inhibits breast cancer growth and metastasis via inducing apoptosis and blocking Src/FAK-involved pathway," Science China Life Sciences, vol. 60, no. 4, pp. 417-428, 2017.
[32] D. Wan, Q. Wu, W. Qu, G. Liu, and X. Wang, "Pyrrolidine dithiocarbamate (PDTC) inhibits DON-induced mitochondrial dysfunction and apoptosis via the NF-kappaB/iNOS pathway," Oxidative Medicine and Cellular Longevity, vol. 2018, Article ID 1324173, 8 pages, 2018.

[33] J. W. Shin, S. B. Kwon, Y. Bak, S. K. Lee, and D. Y. Yoon, "BCI induces apoptosis via generation of reactive oxygen species and activation of intrinsic mitochondrial pathway in H1299 lung cancer cells," Science China Life Sciences, vol. 61, no. 10, pp. 12431253, 2018.

[34] E. Abdelwahid, A. Stulpinas, and A. Kalvelyte, "Effective agents targeting the mitochondria and apoptosis to protect the heart," Current Pharmaceutical Design, vol. 23, no. 8, pp. 1153-1166, 2017.

[35] V. Vijayan, K. Shalini, V. Yugesvaran, T. H. Yee, S. Balakrishnan, and V. R. Palanimuthu, "Effect of paclitaxel-loaded PLGA nanoparticles on MDA-MB type cell lines: apoptosis and cytotoxicity studies," Current Pharmaceutical Design, vol. 24, no. 28, pp. 3366-3375, 2018.

[36] D. Heras-Sandoval, J. M. Pérez-Rojas, J. Hernández-Damián, and J. Pedraza-Chaverri, "The role of PI3K/AKT/mTOR pathway in the modulation of autophagy and the clearance of protein aggregates in neurodegeneration," Cellular Signalling, vol. 26, no. 12, pp. 2694-2701, 2014.

[37] D. D. Sarbassov, D. A. Guertin, S. M. Ali, and D. M. Sabatini, "Phosphorylation and regulation of Akt/PKB by the rictormTOR complex," Science, vol. 307, no. 5712, pp. 1098-1101, 2005.

[38] N. Chu, A. L. Salguero, A. Z. Liu et al., "Akt kinase activation mechanisms revealed using protein semisynthesis," Cell, vol. 174, no. 4, pp. 897.e14-907.e14, 2018. 


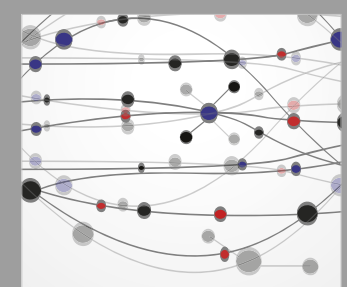

The Scientific World Journal
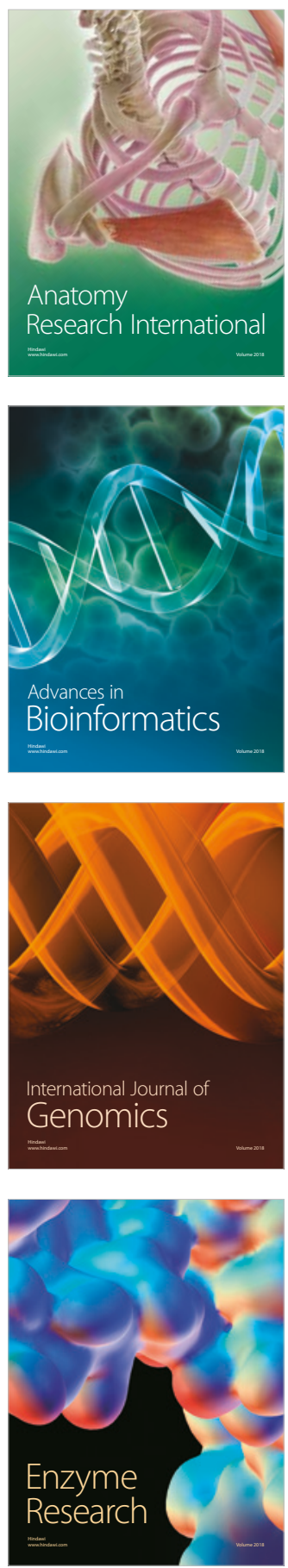
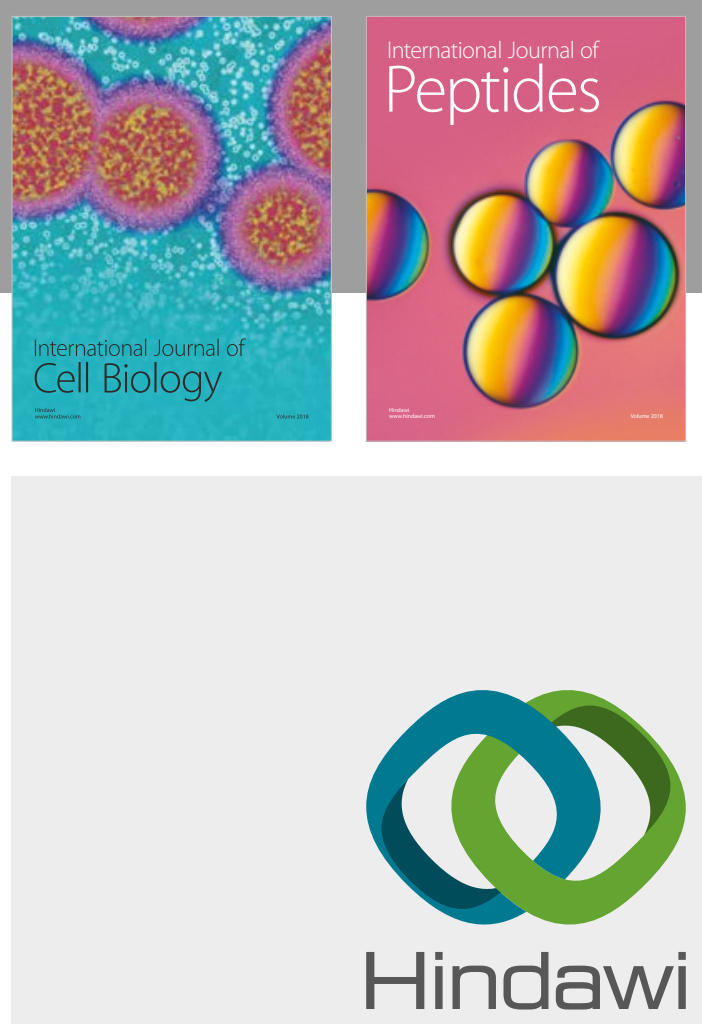

Submit your manuscripts at

www.hindawi.com
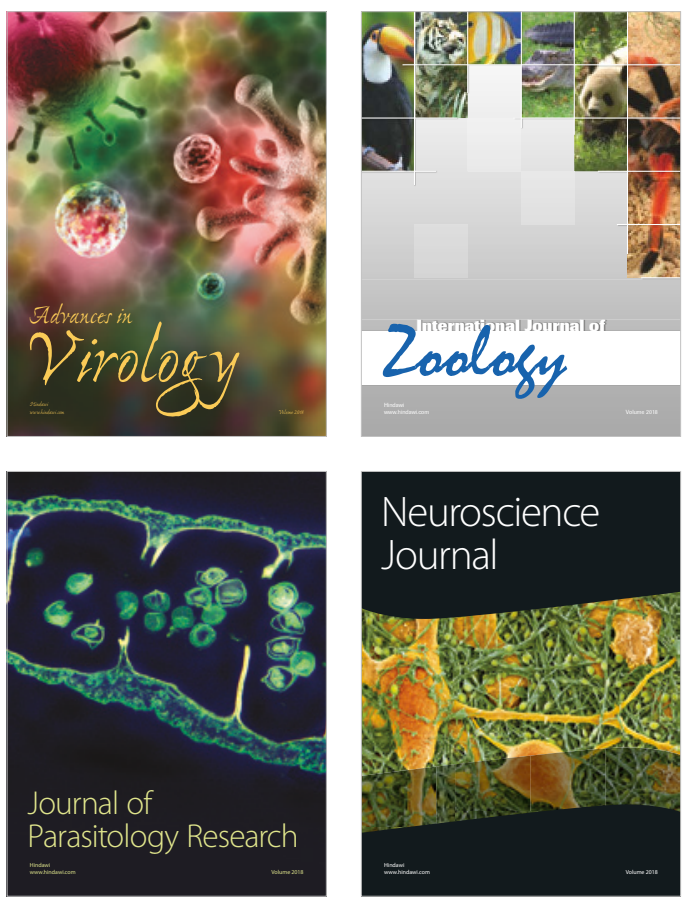
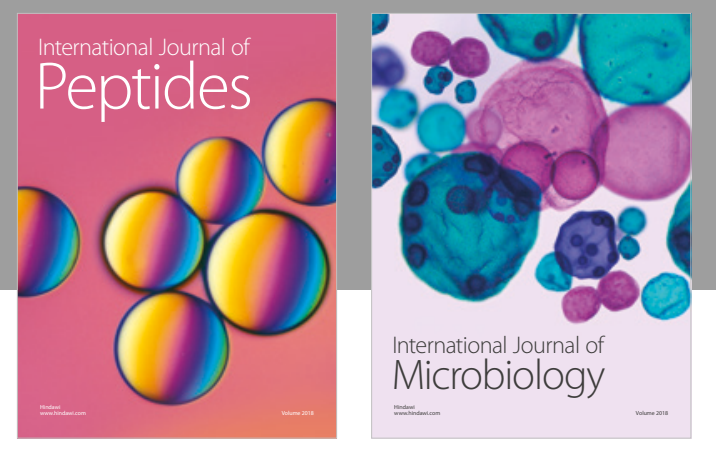

nternational Journal of Microbiology
Journal of
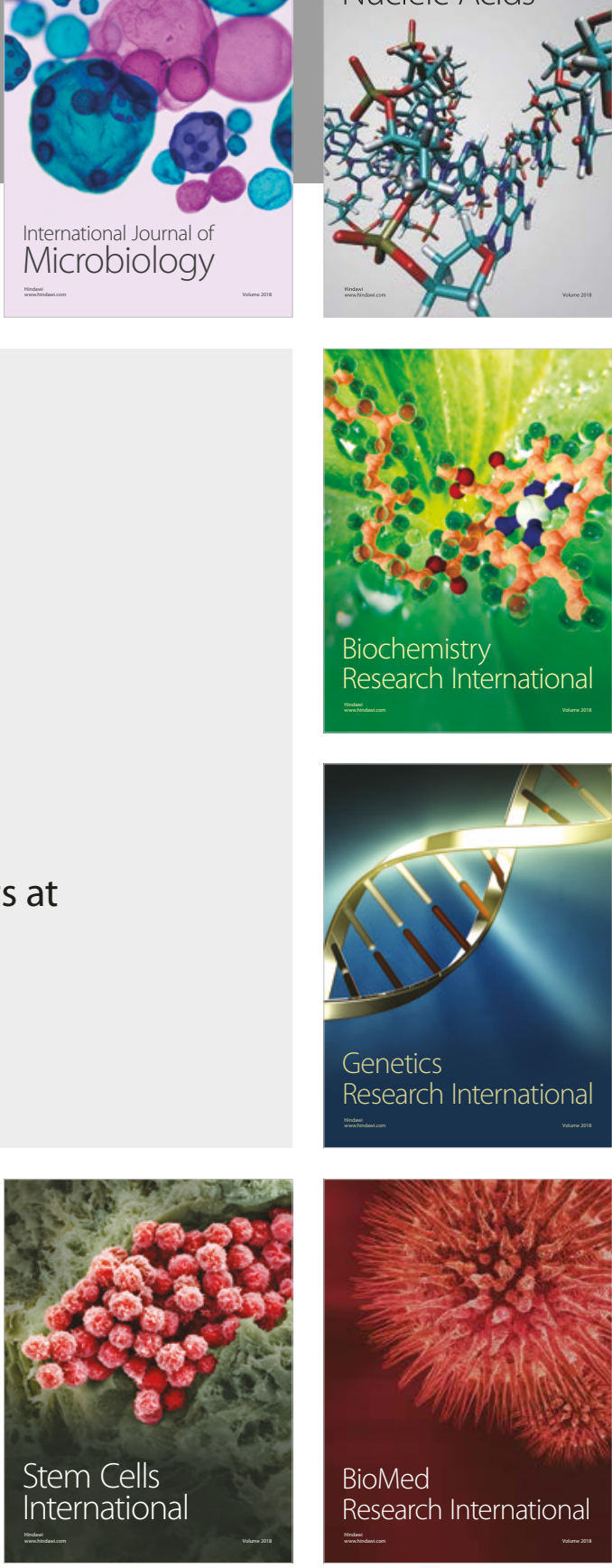
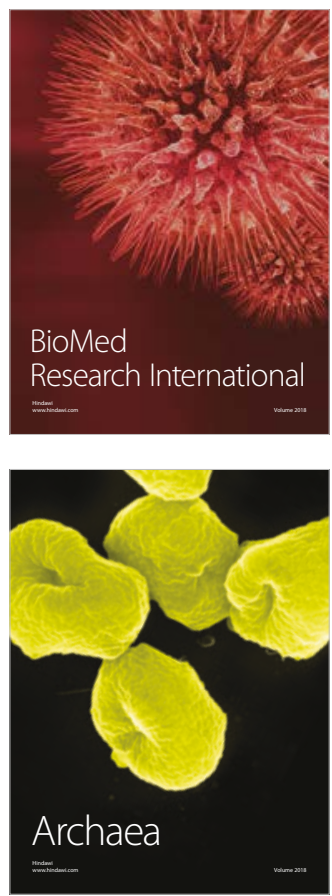